\title{
A Measurement-Based Simulation Model of a Web Cluster Representing SySTEM AND APPlication LEVEL DYNAMiCS
}

\author{
Isabel Dietrich, Kai-Steffen Hielscher, Reinhard German \\ Department of Computer Science 7 \\ University of Erlangen-Nürnberg \\ D-91058 Erlangen, Germany \\ \{isabel.dietrich, ksjh, german\}@informatik.uni-erlangen.de
}

\begin{abstract}
We present a simulation model of a Web cluster which includes important aspects of the hardware, operating and communication systems as well as the application layer for realistic performance evaluations. We formulate the model in UML taking into account both architectural and behavioral aspects and apply detailed system-level measurements at low loads for determining parameters. Measured one-way delays are used to characterize the load on the network. We apply advanced input modeling techniques including multi-modal distributions, multiple phases, and Bézier curves for unconventional shapes to represent all quantities. We also propose a new model based on differences between successive delays for modeling the autocorrelations observed during the measurements. Several internal characteristics of the cluster which significantly affect the behavior at higher loads, including buffering, resource contention, and transport layer mechanisms, are modeled in detail in our UML simulation. The model is able to predict the quantiles of the overall delay for HTTP responses of different configurations beyond the laboratory setup. Replacing all distributions with exponential ones with same means leads to significant deviations, demonstrating the need for serious input modeling.
\end{abstract}

Index Terms-Measurement based simulation, discrete event simulation, input modeling

\section{INTRODUCTION}

Clusters of commodity PC hardware are a popular method to cope with the performance requirements for web servers due to the growing demand for rich media in the web. A lab installation helps us to evaluate the performance implications of this kind of server architectures. We use a measurement infrastructure based on GPS and kernel modifications which allows us to monitor the system at a low level with high precision. This infrastructure is used to observe system parameters such as one-way delays at low loads. According to the nature of the measurements, these delays implicitly include some side-effects such as interrupt latencies and show random behavior.

Emphasis is put to apply advanced input modeling techniques in order to adequately represent these basic parameters. We consider multi-modal delays, as well as phased multi-modal delays, and the inclusion of bézier distributions. We also present a new approach to model auto-correlations observed in the measurements.

We developed a simulation model based on UML constructs which explicitly contains mechanisms such as queuing in buffers, transport control mechanisms, and contention for CPU power with other processes. The model thus combines a precise stochastic representation of low-level system parameters with an explicit representation of system behavior at higher observable levels such as queuing and contention for the CPU.

Equipped with such a fine-tuned detailed model we compare performance figures measured in the actual system with those predicted by the model. The match is good for overall delay quantiles. Replacing the detailed input distributions by exponential distributions with the same mean shows significant differences, thus illustrating the need for serious input modeling.

\section{RELATED WORK}

Performance analyses for web servers are included in the work of numerous authors. Most of them model the system at higher levels of abstraction, e.g. [1]. More detailed simulation models of web clusters are for example included in [2]. Their simulation study is based on a detailed model for the hardware of the system, but does not include fine-grained measurements of delays inside the system. The authors of [3] investigate the effect of different load balancing strategies on clusterbased web servers using both a laboratory cluster and a simulation model. However, they employ only high-level measurements of the load balancer and server service times. In [4] the performance of several load balancing schemes is evaluated. They use traces of the arrival processes as input for their simulation model, but all other aspects of the overall system performance are not based on measurements.

\section{The Web Cluster}

Our laboratory setup includes open-source software for the operating system of the cluster nodes, the web server daemon and the load balancing solution. All software is used on standard off-the-shelf PC hardware.

The Linux Virtual Server [5] system is used for load balancing in our web cluster. The whole distributed web server carries one single IP address called Virtual IP Address (VIP). Requests sent to this address are balanced among the real servers carrying the Real IP Addresses $\left(R I P_{i}\right)$. Using the NAT strategy, incoming packets first arrive at the load balancer node. This node then changes the destination IP address (VIP) in the TCP segments to the IP address of one of the real server nodes $\left(\mathrm{RIP}_{i}\right)$ as determined by the scheduling algorithm. The load balancer is configured as the standard gateway for the reply 


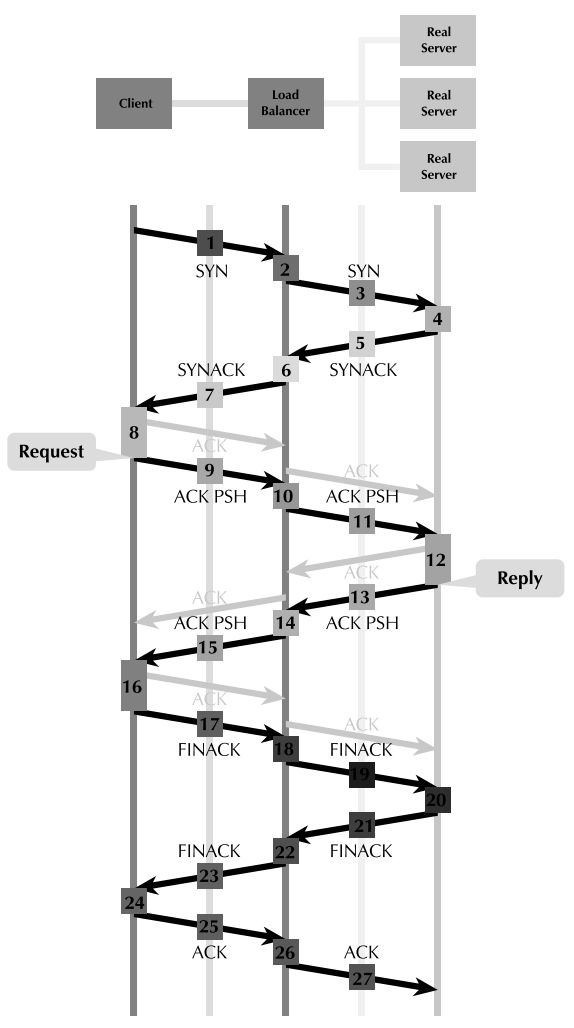

Fig. 1. Illustration of the Delays

packets of the real servers. When packets belonging to replies arrive, the source address is changed to the VIP and the TCP segment is forwarded to the client via the Internet. NAT involves rewriting the packets twice and is limited to geographically close nodes since the load balancer has to be the gateway for all real servers.

\section{MeAsurement InfrastruCture}

Our measurement infrastructure is based on the solution presented in [6], where a software monitoring solution with an extension of the Linux netfilter framework is implemented. Precise measurements of one-way delays are generated using a GPS-based time synchronization solution in combination with NTP [7] and the PPS-API [8]. Some modifications and enhancements of the standard components help to improve timekeeping accuracy. In addition to [6], an offline synchronization solution has been developed [9]. Time stamps of both the events and the PPS pulses are recorded and used to synchronize the clocks after the measurement is over. During the measurement, the system clocks that are used for generating the time stamps are left completely unsynchronized. The offline synchronization after the measurements uses a filtering algorithm to determine and compensate for the variation of the clock frequencies that occurred during the measurement.

On the client side, an HTTP load generator is needed. The tool httperf [10] is able to generate load that overloads a standard web server. The number and the rate of the requests can be specified on the command line. This load generator is ideal for studying the behavior of

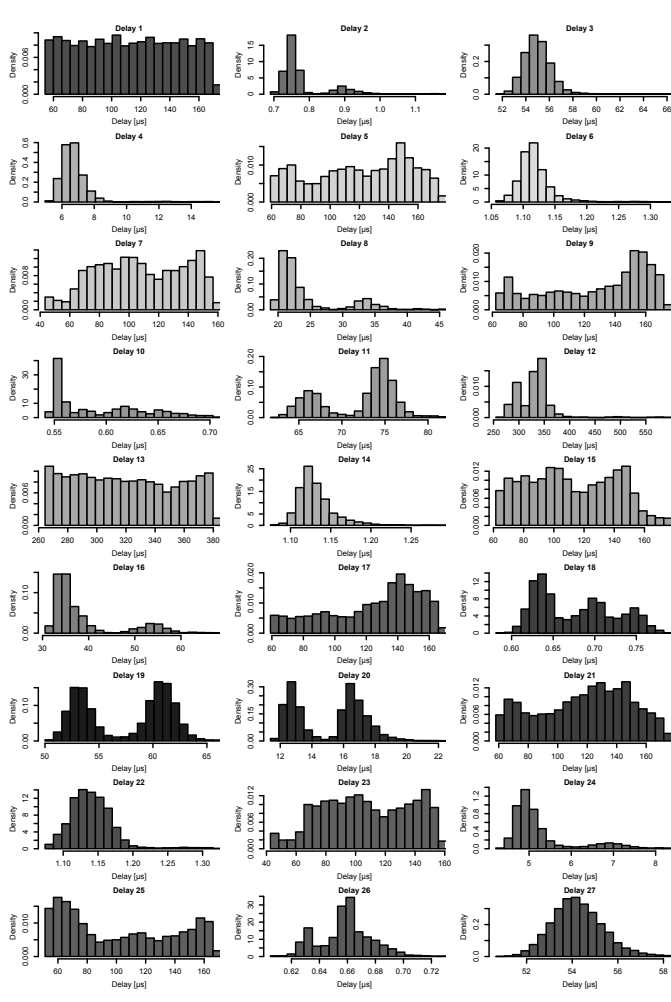

Fig. 2. Histograms of Observed Delays

a web server in extreme situations to find the system's limits. The request generation phase of httperf has been instrumented so that each request on the HTTP layer can be tagged with a time stamp.

\section{Measurements}

For our sample measurements, five real servers and one load balancer were used in a NAT environment with round-robin scheduling. One test client generated HTTP requests using the httperf load generator. We generated 10000 HTTP/1.0 requests for a binary file with a size of 1024 bytes. This resulted in a request size of 65 bytes. The web server added 244 bytes of header information, so the resulting replies had a size of 1268 bytes. Since this is smaller than the maximum segment size used (1500 bytes), all replies consisted of exactly one TCP segment. Fig. 1 illustrates the 27 individual delays occurring in the exchange of TCP segments. Time advances along the vertical axis from top to bottom. The delays, shown as vertical bars, contribute to the total processing time of the HTTP request. The horizontal position shows where the delays are caused: Either by the load generator (LG), the network channel between the load generator and the load balancer $(\mathrm{C} 1)$, the load balancer (LB), the network between the load balancer and the real servers $(\mathrm{C} 2)$ or by one of the real servers (RS).

The delays in the channel $\mathrm{C} 1$ and $\mathrm{C} 2$ include not only the physical propagation delay and the store-and-forward delay of the switches between the hosts, but also the time between the reception of the packet at the node of the 

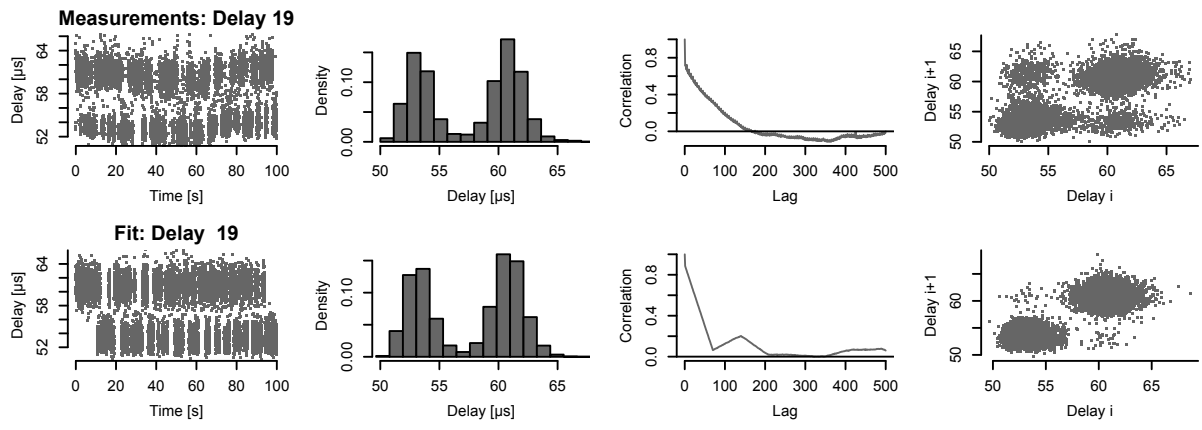

Fig. 3. Delay 19: measured data vs. fitted distribution

cluster and the beginning of the packet processing in the TCP/IP stack of the operating system.

The segments sent during delay 11,12 and 16 are due to TCP protocol mechanisms (fast ACK) and do not mark state changes in the HTTP protocol.

The data gathered in this way enables us to build a detailed input model for a simulation model of our distributed web server and to calibrate and validate the model.

\section{AdVAnCed InPut Modeling}

Fig. 2 depicts the histograms of the 27 measured delays. Each of these delays is used as input for the simulation model. The next sections describe the different possibilities to represent the measured delays in the simulation theoretically.

\section{A. Standard theoretical distributions}

A commonly used method to represent measurements in a simulation are theoretical distribution functions. The distribution fitting tool ExpertFit [11] was used in an attempt to fit theoretical distributions to all 27 delays.

Good fits could be achieved by using this approach for seven delays $(1,4,6,14,22,24,27)$, using mostly the lognormal or log-logistic distributions.

\section{B. Multi-modal delays}

Several of the measured distributions have two or more peaks. This makes it impossible to achieve a good fit with ExpertFit, as the tool is unable to fit distributions to multi-modal data. An easy workaround for this problem is to manually split the data at a threshold and then fit a separate distribution for each part. In the simulation, these separate distributions are combined using the relative frequencies of the respective parts of the data. This method ensures that the weights of the fitted partial distributions approach the weights measured in the real system as the number of sampled delays increases.

The delays 2, 3, 8 and 16 could be fitted in that way.

\section{Phased multi-modal delays}

There are some delays that can not be modeled with the simple multi-modal distributions described above because their peaks are not independent. Inspection of the corresponding trace plots shows that these delays feature alternating phases during which values from the upper or lower parts occur almost exclusively. The top left part of Fig. 3 shows how these phases appear in a trace plot.

The phases have been recreated in the model using a state chart with two main states, one for the phases which contain just values from the upper part, and one for the phases which contain values from the lower part. In each state, delays are sampled exclusively from the distribution associated with that state.

To determine when state changes should occur, approximate minimum phase lengths in seconds for the upper and lower distributions have been identified. One state is active for the duration of the corresponding minimum phase length. Whenever this time has passed, a state change is possible. If the state change really takes place is determined randomly according to the relative frequencies of the measured data parts. This policy ensures that the weights of the fitted distributions approach the measured weights even if the minimum phase lengths behave differently.

For data without a fixed minimum phase length it would be required to fit a distribution for the phase lengths. As we observed a minimum phase length in our measurements, this is not necessary in our model and the presented approach is sufficient.

The delays 10,11, 12, 18, 19, 20 and 26 are represented in this way. Fig. 3 compares the original data and the fit used in the model for delay 19. The trace plot shows that the phases in the simulation approximately match the phases from the measurements. The histograms and also the lag-correlations plots show good matches. The only drawback is that the simulation features less transitions between the peaks than found in the measurements. This is illustrated in the scatter plot on the right. It is probably caused by the fact that the measured phases are not as "clean" as the simulated ones, meaning that lower values sometimes occur in upper phases and upper values in lower phases, resulting in more transitions in the scatter plot.

\section{Bézier distributions}

An alternative to fitting theoretical distributions to data is to use bézier distributions. 

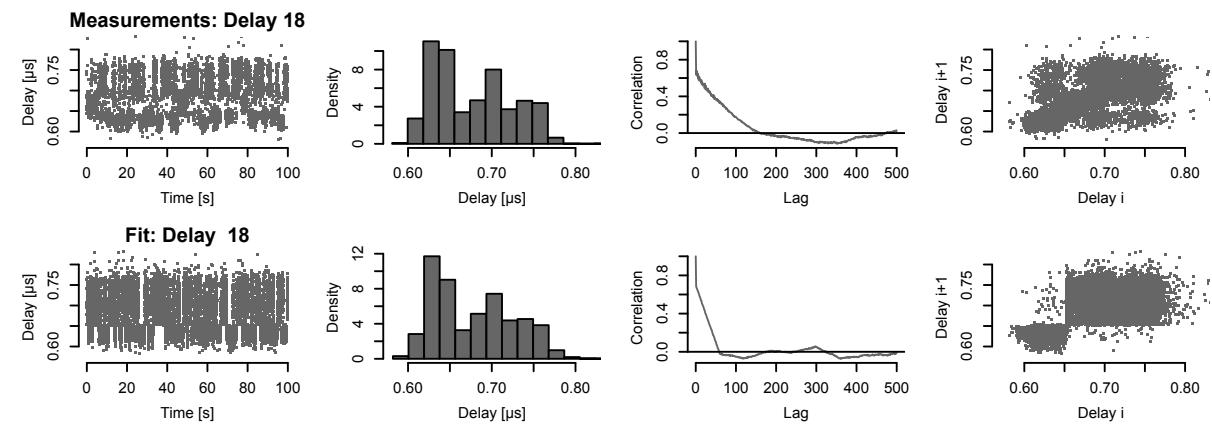

Fig. 4. Delay 18: measured data vs. fitted distribution
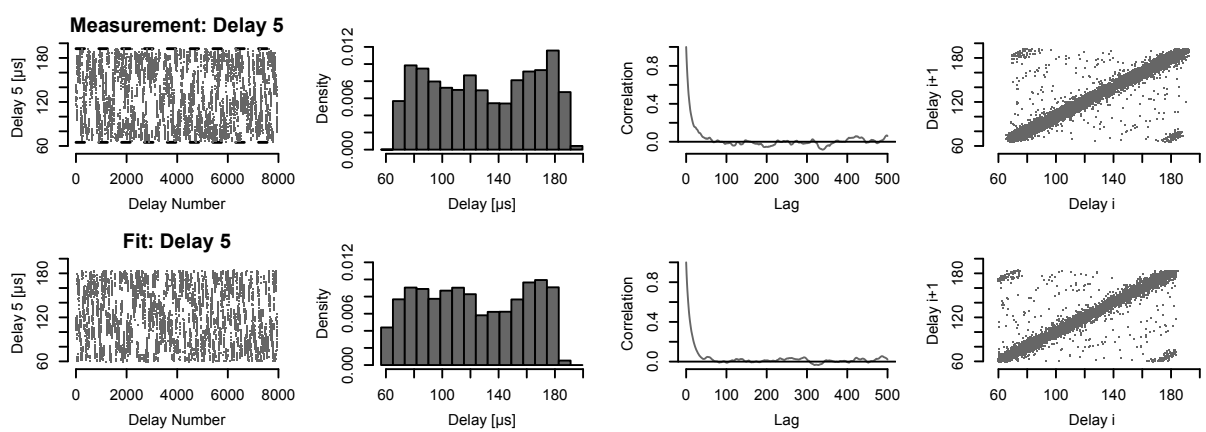

Fig. 5. Delay 5: measured data vs. fitted distribution

The classical bézier curve is a special case of a spline curve, and is often used in computer graphics to approximate smooth univariate functions on a bounded interval. This is done by assigning a number of control points $p_{i}=\left(x_{i}, z_{i}\right)$ through the vicinity of which the curve is bound to pass. A bézier curve with degree $n$ and the control points $\left\{p_{0}, p_{1}, \ldots, p_{n}\right\}$ is given by

$$
P(t)=\sum_{i=0}^{n} B_{n, i}(t) p_{i} \text { for } t \in[0,1],
$$

where the blending function $B_{n, i}(t)$ is the Bernstein polynomial

$B_{n, i}(t)= \begin{cases}\frac{n !}{i !(n-i) !} t^{i}(1-t)^{n-i} & \text { for } t \in[0,1] \\ 0 & \text { and } i=0,1, \ldots, n \\ 0 & \text { otherwise }\end{cases}$

Wagner and Wilson show in [12] how to create a bézier distribution function and how to derive random samples from it with the method of inversion. To create a random sample given a uniformly distributed random number $U$, the first step is to compute $t_{U}$ from the following formula:

$$
U=\sum_{i=0}^{n} B_{n, i}\left(t_{U}\right) z_{i}
$$

To solve this equation, an implementation of a numerical root-finding algorithm is needed. In our simulation model, a combination of two comparably simple algorithms, namely bisection and secant [13] is employed.
First, two approximate values are obtained using two bisection runs of relatively low order (4 and 5). The final solution is computed with the secant method using the bisection results as initial approximations. The random variate $x\left(t_{U}\right)$ can then be delivered according to

$$
x\left(t_{U}\right)=\sum_{i=0}^{n} B_{n, i}\left(t_{U}\right) x_{i}
$$

The graphical tool PRIME, also developed by Wagner and Wilson [12] can be used to fit bézier distributions to sets of custom data. Several automated fitting methods are available as well as the possibility to manually adjust the control points. Result of the fitting process are the control point coordinates.

Three delays, namely 10,18 and 26, are represented by bézier distributions in the model. As the measured distributions are multi-modal, each delay is modeled with two bézier distributions which are combined using the phasing algorithm described above. Fig. 4 shows the fit for delay 18 compared to the measured data. As with the phased multi-modal delays described in the last section, the simulation has less transitions between the phases compared to the measurements. In all other respects, the fitted distribution is a good approximation for the measurements.

\section{A New Model for Autocorrelated DatA}

Nearly all delays in the Fast Ethernet channels (delays $3,5,11,13,19,21,27)$ feature both a high coefficient of auto-correlation over relatively long lags and a clear upper and lower bound (dashed horizontal lines in the 


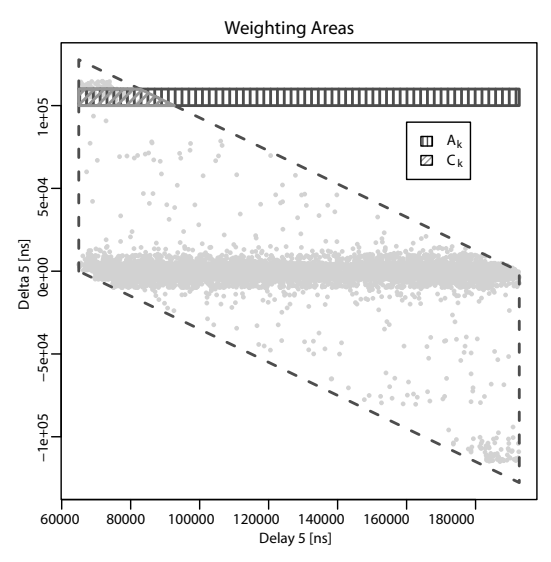

Fig. 6. Valid Sampling Area

top left graph in Fig. 5). The main idea used in our simulation representation is not to sample the delays $d_{i}$ from a distribution directly, but to sample the differences $\delta_{i}=d_{i+1}-d_{i}$ of successive delays. Due to the upper and lower bound $d_{\max }$ and $d_{\min }$ of the delays, there is only a limited range $d_{\min }-d_{i} \leq \delta_{i} \leq d_{\max }-d_{i}$ to sample the deltas from for a given delay $d_{i}$. This valid range can be calculated for all values $d_{\min } \leq d_{i} \leq d_{\max }$. This results in a parallelogram shaped dashed area in Fig. 6, where $\delta$ is plotted over $d$. All points $\left(d_{i}, \delta_{i}\right)$ are located inside the parallelogram.

Inside the valid area, the $\delta_{i}$ are to a large extent independent of the $d_{i}$, as illustrated by the 3-dimensional histogram in Fig. 7.

This fact allows us to construct an overall histogram $H_{o}$ of all $\delta_{i}$ regardless of $d_{i}$. But since only values inside the valid area are used to construct the histogram $H_{o}$, an additional histogram $H_{w}$ is built, where the bins of the histogram $H_{o}$ are weighted with a weighting factor. The factor $w_{k}$ for bin $k$ is calculated as the ratio of the area $A_{k}$ to the area $C_{k} . A_{k}$ is a rectangle delimited on the vertical axis by the borders of bin $k$ and on the horizontal axis by $d_{\min }$ and $d_{\max }$ (see Fig. 6). $C_{k}$ is the portion of this rectangle that is covered by the valid area, i.e. $w_{k}=$ $A_{k} / C_{k}$. For an equidistant histogram with bin width $b$ the area $A_{k}$ is constant: $A_{k}=b \cdot\left(d_{\max }-d_{\min }\right) \forall k$. If $o_{k}$ denotes the number of observations in bin $k$ of $H_{o}$, then the number of observations in bin $k$ of $H_{w}$ is calculated as $o_{k} \cdot w_{k}=o_{k} \cdot A_{k} / C_{k}$.

The resulting histogram $H_{w}$ is the histogram that would be generated, if delta values were observed for all delays $d_{\min } \leq d_{i} \leq d_{\max }$, i.e. even outside of the valid area. The effect of the weighting process is shown in Fig. 8, where the overall histogram $H_{o}$ is overlaid with the weighted histogram $H_{w}$. The histogram $H_{w}$ is used to sample values $\delta_{i}$, which are used to calculate the next delay from the previous one as $d_{i+1}=d_{i}+\delta_{i}$. For this purpose, we sample $\delta_{i}$ from a part of $H_{w}$ that results in a $\delta_{i} \in\left[d_{\min }-d_{i} ; d_{\max }-d_{i}\right]$ for the current delay $d_{i}$. In our example, the bounds for the delay are $d_{\text {min }}=$

64955.5 ns and $d_{\max }=192672.0$ ns. When, e.g., the

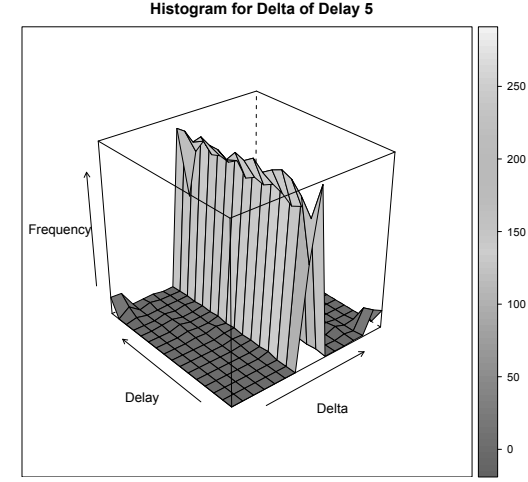

Fig. 7. 3D Histogram of Delta 5

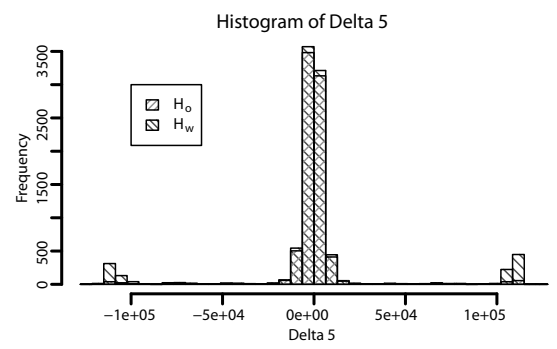

Fig. 8. Original and Modified Histogram of Delta 5

delay $d_{j}$ has reached $190000.0 \mathrm{~ns}, \delta_{j}$ is sampled from the range $\left[d_{\min }-d_{j}=-125044.5 \mathrm{~ns} ; d_{\max }-d_{j}=\right.$ $2672.0 \mathrm{~ns}$. Due to the weighting, a considerable amount of probability mass is found on the extreme negative end of this range and there is a high probability that the delay jumps from a high $d_{j}$ to a low $d_{j+1}=d_{j}+\delta_{j}$ by sampling a low negative $\delta_{j}$. Fig. 5 compares the measured data with values generated using this method. The graphs show a close match of relevant characteristics.

\section{Simulation MOdEL}

The modeling tool AnyLogic [14] was used to build a simulation model of the web cluster. AnyLogic supports discrete event simulation in combination with UML state charts. Our simulation model consists of five top-level building blocks, each of which represents another element of the laboratory cluster. The only means of communication between the five blocks are simulated TCP packets. Fig. 9 illustrates the message flow: simulated requests are generated in the client, then forwarded to the first network channel, processed by the load balancer, and forwarded through the second network channel to the server. The server then processes the received request, and sends one or more reply packets back through the second network channel, the load balancer and the first network channel to the client. The stacked client and server graphics in Fig. 9 indicate that multiple clients and servers are present in the simulation: one client object for every request generated and one server object for every real server in the current simulation setup. 


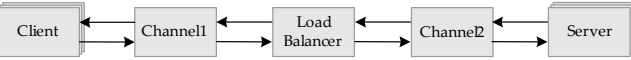

Fig. 9. Conceptual top-level view of the simulation model

Input parameters for the simulation are the distributions for the 27 single delays, the arrival rate of client requests, the sizes of the requested objects, the number of real servers and the load balancing strategy that should be employed. Simulation output data include the 27 simulated delays and the total delay, as well as the utilization, throughput and mean queue length for the network channels, the load balancer and each of the server processors.

\section{A. Client}

The client model evaluates the input parameters and sends HTTP requests to the servers. Its behavior during the communication is governed by a detailed model of TCP. The processing times in the client are modeled according to the input distributions specified above.

Our TCP model supports all important aspects described in the RFCs 793 (basic TCP), 1122 (requirements for Internet hosts), 2581 (congestion avoidance, slow start, fast retransmit) and 2988 (retransmission management). The model can thus deal with packets with arbitrarily large sizes and faulty network channels, just as a real system would. As the measurements were done in a loss-free environment without packet splitting, the mechanisms built into the TCP object provide the possibility to estimate system behavior under different conditions without repeating any measurements.

\section{B. Network channels}

Several aspects had to be paid regard to while modeling the network channels. First, the physical channels are full duplex. This is represented by an independent stream for each direction inside the network objects (Channel 1 and Channel 2 in Fig. 9).

Second, physical channels have a minimum delay that depends (among other factors) on the available bandwidth and the length of the channel. The largest factor contributing to that minimum delay is the transmission delay, i.e. the time needed to feed the packet into the network channel. The other components adding to the network delay (such as propagation delay or queuing delay) are comparably small.

Third, even if the queuing delay is not represented as an absolute value contributing to the minimum channel delay, it still has to be modeled to account for queuing effects at higher utilization levels.

Fourth, the measured delay values have to be accounted for. The measured delays are higher than the sum of transmission and propagation delays because the measurements could not be taken at the exact start and end of the channel, but had to be placed inside the network stacks at the sender and receiver. Thus, the channel delays also represent the nodal processing delay which is caused by the network stack at both ends of the communication and additional hardware inside the channels, like the switch in channel 2. As illustrated in

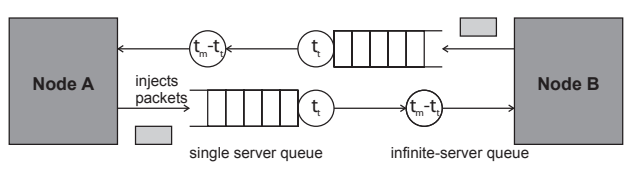

Fig. 10. Concept of the channel representations

Fig. 10, the network channels are assembled as follows: the transmission delay $t_{t}$ is calculated and a single server queue with the transmission delay as required server busy time processes the incoming packet. This also allows for queuing effects at higher utilization levels. Then the packets are processed by an infinite server queue which applies the delays drawn from the distributions fitted above $\left(t_{m}\right)$, reduced by the transmission delay $t_{t}$. The reason for this is that parts of the measured channel delays are not caused by the network, but by the lower parts of the TCP stack where packets are assumed to be processed in parallel.

Channel 1 is modeled as a plain channel with a capacity of one gigabit in each direction. Channel 2 starts with a gigabit channel which leads into a switch where the channel is divided into one 100-megabit channel for each real server.

\section{Load balancer}

The load balancer object distributes the incoming load to the available real servers. The model currently supports three different load balancing schemes: random load distribution, least connection and round robin. Round Robin is the one used in the real system.

\section{Servers}

The server object is the model's most complex building block. It consists of three main parts, the process, system process and processor objects.

1) Process: There is one process object for every single connection to a client. The processes are responsible for processing all incoming packets and sending appropriate reply packets.

2) System Process: The system process object has the sole purpose of occupying the processor from time to time. There are two types of processor allocation demands. The first one occurs quite often, at about the same rate as client requests arrive. This type of system process has to be introduced to model the CPU time consumed by the network stack in the kernel.

The second type of system process represents all the user-mode processes that are running in a real system, require some CPU time, but don't have anything to do with the actual web server processes. They don't occur very often, but require a large amount of processor time, compared to the first type.

The intervals between system processes and the amount of processor time taken each time have been 
calibrated with processor idle data measured in the real system, and with the amount of queuing occurring in the server delays, which is measurable by the number of resulting outlier values.

3) Processor: The reply packets generated by a server process or a system process are handed to the processor object where the actual timekeeping for the server takes place. The processor is modeled as a single server queue. Incoming requests arrive from the process and system process objects. The needed processor time is either specified by the system request, or sampled from the appropriate distributions.

Incoming requests for processor time are divided into requests from user-mode processes and system processes. User-mode processes include all TCP packets carrying data and the second type of requests generated by the system process object. System Processes are the first type of requests from the system process object and TCP packets carrying the SYN or FIN flag. User processes can be interrupted by system processes at any time. This is done to mimic the real system's behavior where longer running processes can and will be interrupted by other processes.

That fact can also be seen while looking at the measurement data, where, at higher request rates, the main server delay has a lot of large outlier values whereas the smaller delays for the SYN and FIN packets still stay within their normal boundaries.

\section{Results}

Several experiments have been conducted with the model. A sequence of five experiments with a request rate increasing from 50 to 1000 requests per second using our detailed input model was used for comparison with the measurement data. Further experiments have been done to compare the performance of our input model to standard exponential distributions. Simulation results were also obtained for a setup with longer reply packets from the servers.

Fig. 11 shows traces, histograms, lag-correlation plots and scatter plots (from left to right) for the different simulation setups and measurements at 100 requests per second. The simulation was stopped as soon as a confidence level of $95 \%$ with the specified relative error was reached after a replication. Although more experiments have been conducted with similar results, a detailed graphical comparison is only presented for the scenario with 100 requests per second due to space limitations.

An important evaluation of our detailed input model consisting of the distributions described in chapters VI and VII is the comparison of the total delays in the simulation and in the measurements. The first and third row of Fig. 11 are relevant for this comparison. They show a good match for the range and magnitude of the delays. Although the simulated mean is slightly larger than in the measurement, the model is still a close representation. The correlation present in the measured data is also represented to some extent.

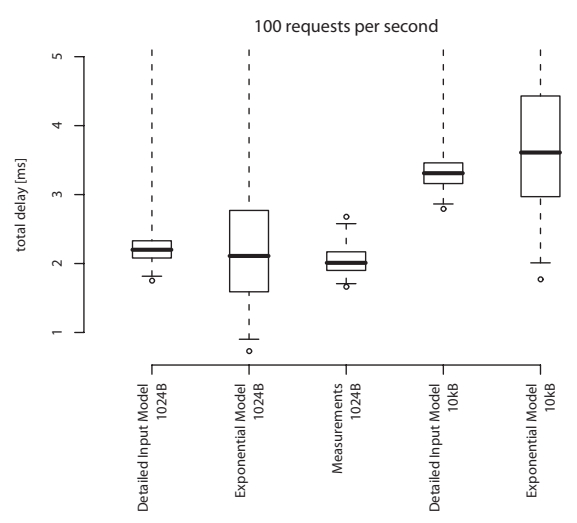

Fig. 12. Quantile comparison for 100 requests per second

Also very important is the question how our input model performs in comparison with a standard model consisting of exponential distributions with the same mean. This comparison is illustrated in the first and second rows of Fig. 11. The measurements, and also our input model, concentrate the total delays on a very narrow range. This is in contrast to the exponential distributions which spread the delays over more than four times the measured range. Due to the nature of exponential distributions, the autocorrelations are not represented. Although the mean total delay gives a good approximation, the overall representation of the measured values is not appropriate.

Fig. 12 compares the quantiles of the total delay for request rate of 100 requests per second in the real cluster and the model in milliseconds. The box plots show the minimum, the $15 \%, 50 \%$ and $85 \%$ quantiles, and the maximum values for the total delay. The plots support the statements above. The median of the exponential distributions seems to be a good approximation, but overall the values are spread too far, which is illustrated by the significant deviations in the $15 \%$ and $85 \%$ quantiles. In contrast, the detailed input model developed by us shows a very good approximation to the measured values.

Further simulation results concern the performance of the system if the requested file size is increased from the standard 1024 bytes to 10 kilobytes. The experimental results are shown in rows four and five of Fig. 11. The corresponding quantiles are also shown in the two rightmost boxes in Fig. 12. Summary measurements taken at the laboratory cluster at 100 requests per second indicate that our detailed input model gives a better approximation for the measured mean and median than the exponential distributions.

\section{Conclusions}

The laboratory setup of the web cluster allows us to conduct fine-grained measurements of the internal behavior in a cluster-based web server. The measurement infrastructure uses GPS, NTP, the PPS-API and extensions like offline synchronization, a kernel buffer for the event trace and an instrumentation of the netfilter code in the Linux kernel. 

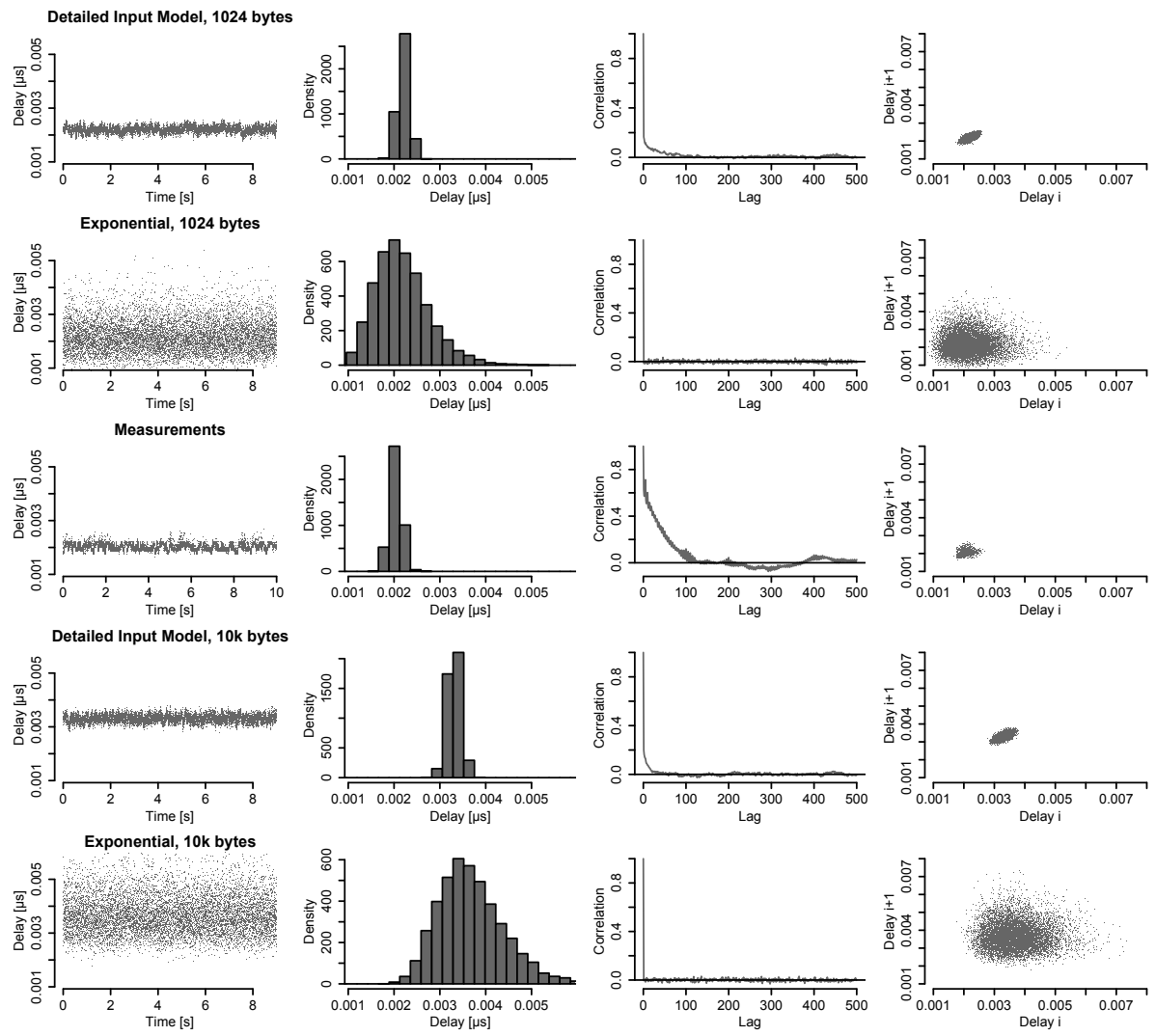

Fig. 11. Traces, histograms and Scatter Plots for the total delays at 100 requests per second

The measured data were built into a detailed UML-based simulation model of the system by combining several input representations like theoretical distributions, multi-modal distributions, phased multi-modal distributions, bézier distributions and a new approach for representing autocorrelated data using the differences between successive delays.

Simulation experiments with a configuration similar to the measurement setup show that the measured delays at different system loads are quite closely reproduced by the model. This enables us to run simulation experiments with various system configurations and system loads, and do a detailed analysis of the estimated system behaviors. Due to the detailed model, the effect of applying even small changes to the system, like modifying the operating system's scheduling and buffering strategies, can be evaluated.

\section{REFERENCES}

[1] D. A. Menascé and V. A. F. Almeida, Capacity Planning for Web Services, 2nd ed. Prentice Hall, 2001.

[2] E. Casalicchio and M. Colajanni, "A client-aware dispatching algorithm for Web clusters providing multiple services," in Proc. of 10th Int'l World Wide Web Conference, May 2001, pp. 535544.

[3] Y. Teo and R. Ayani, "Comparison of Load Balancing Strategies on Cluster-based Web Servers," The Journal of the Society for Modeling and Simulation, vol. 77, no. 5-6, pp. 185-195, November-December 2001.
[4] Q. Zhang, A. Riska, W. Sun, E. Smirni, and G. Ciardo, "Workload-aware load balancing for clustered Web servers," Parallel and Distributed Systems, IEEE Transactions on, vol. 16, no. 3, pp. 219-233, March 2005.

[5] "Linux Virtual Perver Project," http://www.linuxvirtualserver.org/. [Online]. Available: http://www.linuxvirtualserver.org/

[6] K. Hielscher and R. German, "A Low-Cost Infrastructure for High Precision High Volume Performance Measurements of Web Clusters," in TOOLS 2003, Computer Performance Evaluations, Modelling Techniques and Tools, 13th International Conference, Proceedings, P. Kemper and W. Sanders, Eds. Springer, Heidelberg, 2003, pp. 11-28.

[7] D. Mills, "Internet time synchronization: the Network Time Protocol," IEEE Trans. Communications, vol. 39, no. 10, pp. 1482-1493, October 1991.

[8] J. Mogul, D. Mills, J. Brittenson, J. Stone, and U. Windl, "Pulseper-second API for Unix-like operating systems, Version 1," Internet Engineering Task Force, Request for Comments RFC2783, March 2000

[9] K. Hielscher, "Measurement-Based Modeling of Distributed Systems," Ph.D Thesis (Dissertation), University of ErlangenNuremberg, 2008.

[10] D. Mosberger and T. Jin, "httperf: A Tool for Measuring Web Server Performance," in First Workshop on Internet Server Performance. ACM, June 1998, pp. 59-67. [Online]. Available: citeseer.nj.nec.com/mosberger98httperf.html

[11] A. M. Law, "Expertfit distribution fitting software," http://www.averill-law.com/. [Online]. Available: http://www.averill-law.com/

[12] M. A. F. Wagner and J. R. Wilson, "Using univariate Bézier distributions to model simulation input processes," IEEE Transactions, vol. 28, pp. 699-711, 1996.

[13] R. L. Burden and J. D. Faires, Numerical Analysis, 7th ed. Wadsworth Group, 2001.

[14] "Anylogic," http://www.xjtek.com. [Online]. Available: http://www.xjtek.com 